\title{
Nordiques
}

$34 \mid 2017$

La mer Baltique comme zone-frontière : perspectives environnementales, géopolitiques, culturelles

\section{La France et la neutralité finlandaise au temps de la guerre froide}

Nicolas Badalassi

\section{OpenEdition}

\section{Journals}

Édition électronique

URL : https://journals.openedition.org/nordiques/1789

DOI : $10.4000 /$ nordiques. 1789

ISSN : 2777-8479

Éditeur :

Association Norden, Bibliothèque de Caen la mer

Édition imprimée

Date de publication : 1 novembre 2017

Pagination : 115-134

ISBN : 9791095914006

ISSN : $1761-7677$

\section{Référence électronique}

Nicolas Badalassi, «La France et la neutralité finlandaise au temps de la querre froide », Nordiques [En ligne], 34 | 2017, mis en ligne le 25 octobre 2021, consulté le 27 novembre 2021. URL : http:// journals.openedition.org/nordiques/1789; DOI : https://doi.org/10.4000/nordiques.1789 


\section{La France et la neutralité finlandaise au temps de la guerre froide}

Nicolas Badalassi*

\section{RÉSUMÉ}

Jusqu'alors peu étudiée par les historiens, la question de la perception de la neutralité finlandaise par la diplomatie française dans la seconde partie du XX' siècle sinscrit dans un vaste mouvement historiographique visant à réevaluer la place des pays neutres dans les relations internationales après 1945. Si la Finlande ne figure pas parmi les priorités de la politique étrangère de la France au cours de la guerre froide, l'opposition précoce de Paris à la logique bipolaire et son attachement aux principes de l'indépendance et du droit à la neutralité expliquent en grande partie ses manifestations de sympathie et de solidarité à l'égard d'une Finlande qui, dès 1948, tente de rester neutre pour satisfaire son voisin soviétique et éviter de tomber définitivement dans son escarcelle. La fragile position finlandaise provoque ainsi, du côté de la diplomatie française, débats et interrogations quant aux enjeux et à la viabilité de la neutralité dans l'Europe de la guerre froide. Plus encore, l'attention portée à la Finlande reflète systématiquement les préoccupations de la France en matière de sécurité et de coopération européenne. À cet égard, le désir d'indépendance de la France et celui de la Finlande de protéger sa neutralité se rejoignent au début de la décennie 1970 dans un combat commun contre l'interventionnisme soviétique et en faveur du libéralisme politique. En permettant un réel rapprochement entre les deux pays, la Conférence pour la sécurité et la coopération en Europe apparaît comme un tournant.

\section{ABSTRACT}

The issue of the perception of the Finnish neutrality by French diplomacy in the second part of the twentieth century falls within a historiographic movement which aims to reassess the role of the neutral countries in international relations after 1945. Even though the French did not include Finland among the priorities of their foreign policy during the Cold War, Paris' early opposition to bipolarity and its attachment to the principles of independence and right to be neutral mainly explain why it supported Finland which, as soon as 1948, tried to remain neutral to satisfy its Soviet neighbor. The precarious Finnish position thus triggered debates inside the French diplomacy about the stakes of neutrality in Cold War Europe. Moreover, the interest in the situation of Finland systematically reflected French concerns on European security and cooperation. In the early 1970s, the French desire of independence and the willingness of Finland to protect its neutrality came together in a common fight against Soviet interventionism and for political liberalism. The Conference on Security and Cooperation in Europe, by permitting a real rapprochement between Paris and Helsinki, can be considered as a watershed.

\footnotetext{
* Nicolas Badalassi est maître de conférences à l'IEP d'Aix-en-Provence.
} 
La Finlande aurait pu s'abandonner à son destin. Courageusement, elle s'efforce de le dominer et d'échapper au déterminisme, aux fatalités que l'histoire et la géographie semblent devoir lui imposer. Elle a fait, selon la parole de Goethe, de son destin une victoire personnelle. ${ }^{1}$

Ces paroles empreintes d'admiration, prononcées en 1967 par Géraud Jouve, ambassadeur de France en Finlande de 1955 à 1960, renvoient à un point de vue régulièrement exprimé par les diplomates français tout au long de la guerre froide. En effet, des lendemains de la seconde guerre mondiale aux années 1980, la France manifeste un intérêt et une sympathie non dissimulés pour un pays, la Finlande, qui parvient à demeurer neutre tout en étant frontalier de l'Union soviétique. Plus largement, les archives du Quai d'Orsay témoignent de l'attention permanente que la diplomatie française porte à la question de la neutralité nordique au cours de ces décennies.

Un tel intérêt n'a rien de surprenant. Il n'est en réalité que le reflet des interrogations de la France sur sa propre politique étrangère et son rapport à la guerre froide. Quand à partir des années 1945-1946 le conflit est-ouest apparaît inéluctable, le général de Gaulle, qui dirige le gouvernement provisoire jusqu'en janvier 1946, se dit convaincu qu'une division de l'Europe en deux sphères d'influence irait à l'encontre des intérêts européens et français. Cette défiance vis-à-vis de la guerre froide, partagée par une large partie de la classe politique française, s'explique tant par la volonté de maintenir les liens avec Moscou que par le rôle des communistes dans la Résistance puis dans la vie politique du pays. Si l'évolution des rapports est-ouest et le plan Marshall poussent Paris vers l'atlantisme ${ }^{2}$, l'intérêt des Français pour les thèmes de la neutralité et du neutralisme ne faiblit pas et revient régulièrement sur le devant de la scène tout au long de la guerre froide.

Dès le départ, tandis que certains milieux appartenant à la gauche non communiste prônent le "neutralisme positif ", les gaullistes en appellent à une plus grande indépendance à l'égard des deux grands ${ }^{3}$. Le thème de la neutralité n'est ainsi jamais loin, ce qui explique en partie l'attention portée par les uns et les autres aux pays qui, de gré ou de force, suivent cette voie. D'emblée, la neutralité est perçue par les diplomates et responsables français au prisme de leur vision de la politique française et d'une certaine conception de l'intérêt national. En soi, la Finlande est d'une importance stratégique secondaire pour la France, mais

1 Texte de la conférence prononcée par Géraud Jouve, 17 octobre 1967. Archives du ministère français des Affaires étrangères (MAE), Europe 1961-1970, Finlande, 188QO/72.

2 Frédéric Bozo, La politique étrangère de la France depuis 1945, Paris, La Découverte, 1997, p. 6-13.

3 Georges-Henri Soutou, "Les Français et la question d'une éventuelle politique soviétique de "neutralité" en Europe, 1954-1955", in L'URSS et l'Europe, de 1941 à 1957, G.-H. Soutou et E. Robin Hivert (dir.), Paris, PUPS, 2008, p. 428-429. 
sa situation très particulière marquée par une pression incessante de la part de Moscou et un désir de préserver le modèle démocratique et libéral occidental suscite des questionnements permanents de la part d'une diplomatie française soucieuse de garantir et de promouvoir le droit pour chaque pays de mener sa politique étrangère comme il l'entend. La vision française de la neutralité finlandaise reflète ainsi systématiquement les orientations diplomatiques de Paris au cours de la période et conduit les Français à s'interroger sur la place de leur pays dans les relations internationales ainsi que sur la manière de renforcer la sécurité européenne.

Si la question des relations franco-finlandaises demeure encore peu traitée par l'historiographie contemporaine, elle est cependant appelée à se développer dans la mesure où l'accessibilité des sources diplomatiques n'a cessé de s'accroître depuis la décennie 1990 et où le thème de la neutralité et de ses perceptions en période de guerre froide est désormais au centre d'un mouvement de recherche transnational qui vise à réévaluer le rôle des pays neutres dans les relations internationales de la seconde moitié du $\mathrm{XX}^{\mathrm{e}}$ siècle ${ }^{4}$. Les travaux des historiens Jussi Hanhimäki, Jürg Martin Gabriel et Vladislav Zubok ont été précurseurs à plus d'un titre ${ }^{5}$ : en analysant les attitudes américaine et soviétique à l'égard des pays neutres européens, ils ont ouvert la voie à d'autres chercheurs qui ont eux aussi privilégié l'étude des perceptions de la neutralité et du rôle des neutres ${ }^{6}$. Une attention particulière est portée aux cas finlandais, suédois, autrichien et suisse, mettant en avant les différences qui les séparent, que ce soit sur la nature et l'origine de leur neutralité ou leurs liens avec les deux blocs. Ainsi, alors que la Suède et la Suisse ont fait de la neutralité le pivot de leur politique étrangère depuis 1815, la neutralité de la Finlande et de l'Autriche découle de facteurs contraignants (traité d'État autrichien de 1955, voisinage soviétique pour la Finlande).

4 Neutrality and Neutralism in the Global Cold War. Between or Within the Blocs?, S. Bott, J. M. Hanhimäki, J. M. Schaufelbuehl et M. Wyss (dir.), Londres, Routledge, 2015 ; Journal of Cold War Studies, vol. 18, no 3, Neutrality and World Politics during the Cold War, J. Aunesluoma, T. C. Fischer et A. Makko (dir.), 2016. La perception française de la neutralité nordique durant le premier $\mathrm{XX}^{\mathrm{e}}$ siècle a été bien analysée ; voir par exemple : Louis Clerc, «The Hottest Places in Hell ? Finnish and Nordic Neutrality from the Perspective of French Foreign Policy, 1900-1940", in Caught in the Middle. Neutrals, Neutrality and the First World War, J. den Hertog et S. Kruizinga (dir.), Amsterdam, Amsterdam University Press, 2011, p. 139-153.

5 Jussi M. Hanhimäki, Containing Coexistence. America, Russia, and the "Finnish Solution", 1945-1956, Kent, Kent State University Press, 1997 ; id., An Insecure Friendship. The United States and Scandinavia since 1945, Londres, Palgrave Macmillan, 1997 ; Jürg Martin Gabriel, The American Conception of Neutrality after 1941, Londres, Palgrave Macmillan, 1988 ; Vladislav Zubok, "The Soviet Attitude towards the European Neutrals during the Cold War ", in Die Neutralen und die europäische Integration 1945-1995, M. Gehler et R. Steininger (dir.), Vienne, Böhlau, 2000, p. $29-43$.

6 Aryo Makko, "Sweden, Europe, and the Cold War. A Reappraisal ", Journal of Cold War Studies, vol. 14, no 2, 2012, p. 68-97 ; Neutrality in Austria, G. Bischof, A. Pelika et R. Wodak (dir.), Londres, Taylor \& Francis, 2001. 
De nombreux travaux issus de l'historiographie récente s'interrogent par ailleurs sur la viabilité du statut de pays neutre dans l'Europe post-1945, faisant valoir la nature idéologique de la guerre froide : parce que le conflit est-ouest, avant tout politique, dépasse la simple logique interétatique et les aspects purement militaires de la stratégie, la question des limites de la neutralité se pose. Dans ses articles sur la Suisse, Georges-Henri Soutou met en évidence la différence entre neutralité et neutralisme, soulignant que la neutralité n'est ni politique ni économique mais strictement militaire, alors que le neutralisme consiste à ne se rapprocher ni d'un bloc ni de l'autre, quel que soit le domaine concerné. Il montre ainsi que si la Suisse est toujours restée attachée à sa neutralité, elle n’a en rien fait preuve de neutralisme dans la mesure où son modèle économique et ses idées libérales la rapprochaient naturellement des pays de l'OTAN ${ }^{7}$.

Pour ce qui est de la Finlande, où là aussi la neutralité l'emporte sur le neutralisme, ce sont, sans surprise, les historiens finlandais qui sont les plus prolixes. Les travaux de Rinna Kullaa, Johanna Rainio-Niemi, Juhana Aunesluoma ou Kimmo Rentola montrent comment la neutralité est devenue partie intégrante de l'identité de la Finlande, quelle a été la place de ce pays dans la politique étrangère de l'URSS et, inversement, quelle a été l'importance du facteur soviétique dans la politique extérieure finlandaise ${ }^{8}$.

Les recherches récentes mettent par ailleurs en exergue certains moments de la guerre froide plus que d'autres, pendant lesquels le rôle des neutres d'Europe a été central. Cela est particulièrement le cas pour la fin des années 1960 et la première moitié des années 1970. Durant cette période, la Finlande, la Suède, l'Autriche, la Suisse, mais également la Yougoslavie sont au premier plan des relations internationales en Europe : portant à bout de bras le vieux projet soviétique de conférence sur la sécurité européenne qui doit, pour le Kremlin, rendre pérenne la division du continent en blocs, les neutres contribuent largement à transformer ce qui est devenu la Conférence pour la sécurité et la coopération en Europe (CSCE) en outil de dépassement de la guerre froide. Adoptant un " neutralisme actif» qui dissimule en réalité un soutien assez net aux positions libérales occidentales sur les droits de l'homme, les échanges culturels ou la circulation des personnes, les neutres prouvent leur capacité à utiliser les leviers

7 Georges-Henri Soutou, "La France et la Suisse au XXe siècle : de la méfiance stratégique à la confiance et à la complicité ", Stratégique, 107, 4, 2014, p. 17-33.

8 Rinna Kullaa, Non-Alignment and its Origins in Cold War Europe. Yugoslavia, Finland and the Soviet Challenge, Londres - New York, I.B.Tauris, 2012 ; Juhana Aunesluoma, Johanna Rainio-Niemi, "Neutrality as Identity : Finland's Quest for Security in the Cold War ", Journal of Cold War Studies, vol. 18, no 4, 2016, p. 51-78; Kimmo Rentola, Niin kylmää että polttaa. Kommunistit, Kekkonen ja Kreml 1947-1958, Helsinki, Otava, 1997. 
multilatéraux qui s'offrent à eux pour prendre une part active au renforcement de la sécurité et de la coopération en Europe. Ils donnent ainsi la preuve de leur aptitude à intervenir dans les affaires du continent sans pour autant être membres de l'une ou l'autre des alliances militaires européennes. De fait, l'historiographie présente leur rôle pendant la CSCE - ou " processus d'Helsinki » - comme leur plus haut fait d'armes au cours de la guerre froide'. La quantité impressionnante de sources consacrées au rôle des neutres lors de cette conférence témoigne à elle seule de l'importance de cet épisode. Si on considère les seules archives françaises, on s'aperçoit que le nombre de documents du Quai d'Orsay portant sur la place des neutres dans les rapports est-ouest augmente sensiblement à partir de 1969. La Finlande est incontestablement celui des quatre pays cités précédemment qui attire le plus l'attention des diplomates français, à tel point que le début des années 1970 apparaît comme une véritable rupture à la fois dans la manière dont Paris perçoit Helsinki et dans les rapports entre les deux pays. Le dynamisme finlandais au moment de la CSCE fait l'objet de très nombreux télégrammes, notes et rapports qui, plus encore que pour les années qui précèdent, laissent entrevoir un réel intérêt de la France pour la situation de la Finlande et son engagement dans les relations internationales en Europe. C'est ce foisonnement de sources à partir de la fin des années 1960 qui explique d'une part l'impression d'un déficit d'informations sur la Finlande avant la décennie 1970 et d'autre part l'insistance sur la fin de la période de l'exposé qui suit.

Néanmoins, il convient de garder à l'esprit que, même pour la décennie 1950, les archives du Quai d'Orsay relatives à la Finlande sont les plus fournies parmi celles consacrées aux pays neutres européens (sans compter la Suisse, pays frontalier de la France). Rappelons que, sous la Cinquième République, la politique étrangère est l'un des domaines réservés au président de la République, chargé d'en définir les grandes orientations. Le ministère des Affaires étrangères a pour mission de la mettre en œuvre. Il est divisé en directions pour la plupart géographiques (Europe, Amérique, Asie...), elles-mêmes subdivisées en sous-directions. L'ambassade de France à Helsinki est ainsi rattachée à la sous-direction d'Europe occidentale, ce qui prouve que, malgré son statut de neutre et l'influence soviétique, la Finlande est vue par Paris comme faisant partie intégrante de l'Occident. Les ambassades rendent compte notamment des évolutions politiques, diplomatiques et économiques de chaque pays, permettant aux directions et sous-directions de formuler des orientations politiques précises à l'égard de tel ou tel ensemble géographique ou domaine d'intervention. Des directives sont ainsi

9 Thomas Fischer, Neutral Power in the CSCE. The N+N States and the Making of the Helsinki Accords 1975, Baden-Baden, Nomos, 2009. 
quotidiennement transmises par les directions centrales aux ambassades pour garantir la cohérence de la politique extérieure française ${ }^{10}$.

Pour ce qui est des documents qui figurent dans les cartons "Finlande ", ils sont la plupart du temps produits par l'ambassade de France à Helsinki ou dans les ambassades françaises à Stockholm et à Moscou, rarement par l'administration centrale. Ce constat prouve bien que la Finlande reste périphérique dans la définition de la politique étrangère de la France et que seuls les diplomates en poste en Europe du Nord développent une réflexion à long terme quant à la place de ce pays dans le conflit est-ouest. Ils se cantonnent souvent aux questions purement politiques et diplomatiques. Toutefois, là encore, on note une sensible évolution à partir de la fin des années 1960, lorsque le gouvernement finlandais se fait le promoteur du projet de conférence sur la sécurité européenne. La direction d'Europe et celle des Affaires politiques du Quai d'Orsay considèrent alors avec plus d'attention les avis exprimés par l'ambassadeur de France en Finlande - Gérard André (1969-1975) puis Jacques Chazelle (1975-1981) - lorsqu'elles élaborent les positions françaises vis-à-vis de la détente est-ouest et de la CSCE.

Reste que, on l'a dit, les relations franco-finlandaises après 1945 n'ont pas fait l'objet d'une étude approfondie et demeurent un domaine inexploré de l'historiographie récente. Les quelques pistes posées ici cherchent précisément à mettre en lumière ce chantier prometteur, notamment au vu des documents d'archives français, sur lesquels se fonde cette courte présentation. Il y a fort à parier que les fonds d'archives finlandais recèlent des informations qui permettraient de dresser un panorama complet des relations entre les deux pays et de comprendre comment les Finlandais présentent leur neutralité et s'efforcent, de leur côté, de la faire correspondre à des évolutions de la politique étrangère française. L'analyse de ces documents ne pourrait qu'enrichir nos connaissances des rapports intra-européens et de la politique étrangère de la France pendant la guerre froide, dont les aspects les plus parcourus par les historiens ont rarement trait à l'Europe nordique.

En effet, si les recherches sur la politique extérieure française après 1945 se sont considérablement développées depuis la fin du $\mathrm{XX}^{\mathrm{e}}$ siècle du fait de la facilité d'accès aux documents d'archives - français ou non -, elles ont privilégié les relations de la France avec les deux géants américain et soviétique, avec ses voisins européens et méditerranéens, avec les pays d'Europe de l'Est ou avec les organisations internationales, permettant d'identifier les continuités et les ruptures qui ont marqué les décennies écoulées depuis la fin de la seconde guerre

10 Sur le fonctionnement du Quai d'Orsay, voir : François Plaisant, Le ministère des Affaires étrangères. La diplomatie des années 2000, Paris, Milan, 2000. Sur la diplomatie française en temps de guerre froide, voir le chapitre 6 de l'ouvrage de Maurice Vaïsse, La grandeur. Politique étrangère du général de Gaulle, 1958-1969, Paris, Fayard, 1998. Sur les pratiques des diplomates français, voir Christian Lequesne, Ethnographie du Quai d'Orsay. Les pratiques des diplomates français, Paris, CNRS Éditions, 2017. 
mondiale ${ }^{11}$. Elles ont souligné par exemple que la volonté de préserver une certaine marge de manœuvre vis-à-vis des États-Unis et de maintenir le dialogue avec l'URSS n'est pas apparue avec l'avènement de la République gaullienne mais a émergé dès les dernières années de la Quatrième République ; le général de Gaulle, une fois revenu au pouvoir, n'a fait que reprendre cette idée en lui conférant une dimension et un éclat plus importants. Ce pilier de la politique étrangère française qu'est l'attachement à l'indépendance de la France est donc né avec le déplacement du centre de gravité des relations internationales vers Moscou et Washington, mais il s'est imposé de manière plus claire à partir des années 1960. L'intérêt de la France pour la Finlande et sa neutralité a suivi le même chemin : présent mais timoré en début de période, il est affirmé sans ambages durant les années de détente. Ce parallèle est d'autant plus évident que, sans jamais devenir neutre, la France n'a pas cessé de voir dans la neutralité un statut à défendre face à la course aux territoires menée par les États-Unis et l'URSS à travers la planète. Ainsi, le 25 janvier 1974, le représentant de la France à la CSCE propose d'inclure dans la partie du futur Acte final d'Helsinki consacrée à «l'égalité souveraine et $[\mathrm{au}]$ respect des droits inhérents à la souveraineté » l'idée selon laquelle chaque État a le droit «d'appartenir ou de ne pas appartenir à des organisations internationales, d'être partie ou non à des traités bilatéraux ou multilatéraux, y compris le droit d'être partie ou non à des traités d'alliance $»^{12}$. De cette façon, la diplomatie française cherche non seulement à consacrer l'un des principes phares de la politique gaullienne, mais également à garantir la sécurité des pays neutres ou non alignés, et en particulier de la Finlande, soumise à la pression permanente du grand voisin soviétique. La CSCE apparaîtrait à cet égard comme l'aboutissement d'une volonté française, amorcée dès les prémices de la guerre froide, de protéger les neutres et l'idée même de neutralité. L'examen de la perception française de la neutralité finlandaise révèle pourtant un cheminement moins linéaire et il est clair que la détermination française à défendre la Finlande et les neutres a varié en fonction de l'évolution des relations internationales et des priorités et intérêts de la politique extérieure de la France. Ce sont les évolutions de cette perception entre l'ère khrouchtchévienne et le début des années 1980 que nous allons tenter de présenter ici.

11 Voir par exemple : Frédéric Bozo, La politique étrangère de la France depuis 1945, op. cit. ; MariePierre Rey, La tentation du rapprochement. France et URSS à l'heure de la détente, Paris, Presses de la Sorbonne, 1991 ; Thomas Gomart, Double détente. Les relations franco-soviétiques de 1958 à 1964, Paris, Publications de la Sorbonne, 2003 ; Frédéric Bozo, Deux stratégies pour l'Europe. De Gaulle, les ÉtatsUnis et l'Alliance atlantique (1958-1969), Paris, Plon, 1996 ; Jenny Raflik-Grenouilleau, La Quatrième République et l'Alliance atlantique. Influence et dépendance (1945-1958), Rennes, Presses universitaires de Rennes, 2013 ; Gérard Bossuat, La France et la construction de l'unité européenne, de 1919 à nos jours, Paris, Armand Colin, 2012.

12 Documents CSCE/II/A/16, 25 janvier 1974 et CSCE/II/A/21, 31 janvier 1974. Archives CSCEPrague, Book 8, Helsinki 1972-1975. 


\section{LES ENJEUX DE LA NEUTRALITÉ À L'Ère KHROUCHTCHÉVIENNE}

L'aversion précoce des dirigeants français à l'égard de la " politique des blocs " explique l'anxiété avec laquelle ils perçoivent les tentatives du Kremlin d'étendre son influence en Europe septentrionale à la fin des années 1940. La signature du traité d'amitié fenno-soviétique le 6 avril 1948, grâce auquel la Finlande évite de peu la soviétisation mais s'engage à demeurer neutre, est ainsi l'une des premières occasions pour la diplomatie française de se pencher sur la problématique de la neutralité en temps de guerre froide ${ }^{13}$. Mais c'est la période qui court de la mort de Staline à la chute de Nikita Khrouchtchev qui, en faisant de la neutralité un thème essentiel des rapports est-ouest, conduit la France à porter un intérêt soutenu à cette question et, de fait, à la neutralité finlandaise.

La question de l'extension de la neutralité en Europe est en effet au cœur du dialogue est-ouest tout au long des années 1950. Dès 1952, dans l'espoir de régler la question allemande dans un sens favorable aux intérêts soviétiques, Staline suggère de neutraliser l'Allemagne. Cette idée, qui figure également dans le Traité de sécurité collective en Europe proposé par le ministre soviétique des Affaires étrangères Viatcheslav Molotov aux Occidentaux en 1954, devient par la suite l'un des piliers de la stratégie khrouchtchévienne de coexistence pacifique. Le principe d'une neutralisation progressive de l'Europe centrale puis septentrionale à partir de l'Allemagne, qui à partir de la révolte des ouvriers de Berlin-Est en 1953 reflète la crainte soviétique de l'influence occidentale à l'Est, se retrouve dans les différents plans de sécurité européenne proposés par l'URSS et la Pologne aux pays de l'OTAN à la fin des années 1950 et au début des années 1960 (plans Rapacki, Khrouchtchev et Gomulka). L'objectif de Moscou est clair : la neutralisation, conjuguée à la création d'organismes de sécurité collective, doit permettre de distendre les liens entre l'Europe occidentale et les États-Unis et ainsi d'accroître l'influence soviétique sur le continent ${ }^{14}$. La Finlande apparaît comme l'exemple à suivre et Khrouchtchev n'hésite pas à l'utiliser pour montrer qu'il est un homme de bonne volonté. Non seulement il affirme publiquement son soutien à la neutralité de ce pays, mais il lui rétrocède en septembre 1955 la base de Porkkala, occupée par l'Armée rouge depuis 1944, et l'autorise à intégrer le Conseil nordique ${ }^{15}$.

13 Nicolas Badalassi, "France, the European Neutrals and the Kremlin, 1947-1981 ", in European Neutrality and the Soviet Union during the Cold War, M. Kramer, A. Makko et P. Ruggenthaler (dir.), Lanham, Lexington Books, à paraitre.

14 Nicolas Badalassi, En finir avec la guerre froide. La France, l'Europe et le processus d'Helsinki, Rennes, PUR, 2014, p. 48-57.

15 Sabine Dullin, "Où se trouve la frontière ? La place de la Finlande dans la zone de sécurité de l'URSS, 1944-1956 ", in L'URSS et l'Europe, de 1941 à 1957, op. cit., p. 376. 
Si les plans soviéto-polonais de sécurité européenne suscitent des débats dans un Quai d'Orsay largement dominé par les adversaires de la neutralisation de l'Allemagne, la diplomatie française est en revanche unanime quant aux intentions de Moscou à l'égard de la Finlande. Le renforcement de son statut de pays neutre fait partie intégrante de la propagande soviétique : Khrouchtchev veut " utiliser le porte-panneau réclame finlandais et se servir de la Finlande pour montrer aux Scandinaves l'intérêt qu'il y a à mériter [sa] confiance ${ }^{16}$. Paris n'est donc pas dupe et considère la neutralité finlandaise comme relevant de problématiques distinctes du reste de l'Europe.

Le regard français sur la Finlande acquiert pourtant une dimension nouvelle à partir du moment où le président finlandais Urho Kekkonen profite de la restitution de Porkalla pour " donner plus de substance à l'idée de neutralité ${ }^{17}$ et ainsi prouver que son pays peut être vraiment neutre. Les archives du Quai d'Orsay consacrées à la Finlande sont éloquentes à cet égard : la quasi-totalité des cartons porte sur la politique de neutralité, surtout lorsque celle-ci est de nouveau menacée par Moscou à partir de 1957 sous prétexte d'un imminent réarmement allemand. En effet, à cette date, les progrès nucléaires soviétiques et le lancement du Spoutnik conduisent les Occidentaux à vouloir équiper les armées européennes d'armes nucléaires tactiques. Aux yeux de Khrouchtchev, le fait que la Bundeswehr soit elle aussi concernée par une telle mesure apparaît comme une véritable provocation. Face à cette menace, le Premier secrétaire du PCUS cherche à garantir la sécurité des frontières soviétiques et, par conséquent, lance une offensive diplomatique en direction de la Finlande, dénonçant l'action néfaste des "milieux réactionnaires et des dirigeants socialistes finlandais ", jugés trop favorables à l'Occident ${ }^{18}$. Il obtient rapidement la démission du président socialiste du Conseil et la constitution d'un cabinet agrarien homogène et minoritaire avalisée par Kekkonen. En échange, le dirigeant soviétique consent à ouvrir des négociations avec la Finlande " en vue de l'établissement d'un protocole d'échanges commerciaux pour 1959 et la conclusion d'un nouvel accord commercial à long terme $»^{19}$. Pour l'ambassadeur français à Moscou Maurice Dejean, le geste de Khrouchtchev prouve sa crainte d'une réorientation vers l'Ouest de la politique étrangère finlandaise par les socialistes finnois ; il est aussi un moyen pour le Kremlin de faire savoir à la Finlande qu'elle doit se tenir à l'écart des divers organismes de coopération européenne. Cependant, estime l'ambassadeur français, l'URSS, en agissant de la sorte à l'encontre d'un État neutre, "risque

16 Télégramme de Jacques Lecompte-Boinet, 22 juillet 1954. MAE, Europe 1944-1960, Finlande, 34.

17 Rapport de B. Dufournier, 3 juin 1967. MAE, Europe 1961-1970, Finlande, 188QO/72.

18 Conférence de Géraud Jouve, 17 octobre 1967. MAE, Europe 1961-1970, Finlande, 188QO/72.

19 Télégramme de M. Dejean, 29 janvier 1959, in Documents diplomatiques français, Paris, La Documentation française, 2003, p. 116-120. 
d'éveiller la méfiance dans les États que la propagande soviétique s'efforce de séduire ", à commencer par les pays scandinaves ${ }^{20}$.

L'offensive soviétique ne cesse pas pour autant. Le 30 octobre 1961, l'URSS adresse une note à la Finlande en invoquant l'article 2 du pacte d'amitié fennosoviétique de 1948 qui stipule que " les hautes parties contractantes se consulteront dans le cas où l'on constaterait la menace d'une agression militaire ». Le prétexte soviétique est que le réarmement de la RFA et les accords de celle-ci avec le Danemark et la Norvège - notamment en matière de sécurité maritime constituent un danger d'agression contre l'URSS à travers le territoire finlandais. Si Khrouchtchev finit par renoncer aux consultations militaires - Kekkonen l'ayant persuadé que cela ne ferait que jeter l'inquiétude dans les pays scandinaves voisins -, Dejean n'en considère pas moins cet ajournement comme provisoire, estimant que « sa durée dépend de l'évolution de la situation dans l'ensemble de l'Europe du Nord et de la Baltique ${ }^{21}$. En d'autres termes, un renforcement effectif de la coopération militaire entre la RFA et le Danemark en particulier pourrait aboutir à une nouvelle offensive soviétique à l'égard d'Helsinki.

Dejean s'inquiète également des manœuvres du Kremlin visant à influencer la politique commerciale de la Finlande : tandis que celle-ci devient membre associé de l'Association européenne de libre-échange (AELE) en 1961, Khrouchtchev ne tolère un tel rapprochement qu'à condition que le gouvernement finlandais applique à l'URSS la clause de la nation la plus favorisée. Pour l'ambassadeur, en abaissant de $40 \%$ ses droits de douane vis-à-vis de Moscou et ce sans réciprocité, Helsinki accroît sa dépendance à l'égard de son puissant voisin, affaiblit la portée de l'AELE - ce qui n'est pas pour déplaire à la France gaullienne dans la mesure où l'AELE est d'abord un outil des Britanniques pour contrebalancer la CEE - et, surtout, crée un précédent « qui risque de renforcer un jour l'hostilité de Moscou à l'égard des pays neutres qui demandent leur association au Marché commun " ${ }^{22}$. Dejean voit juste : durant les mois et les années qui suivent, l'Autriche subit les foudres du Kremlin à partir du moment où elle négocie avec Bruxelles en vue d'une association à la CEE.

Face à ces tentatives soviétiques d'ébranler la neutralité finlandaise et à l'heure où, à Paris, le général de Gaulle - revenu au pouvoir en 1958 à la faveur de la crise algérienne - se pose en défenseur du droit des peuples à l'indépendance et en pourfendeur de la politique des blocs, l'ancien ambassadeur de France à Helsinki de 1955 à 1960, Géraud Jouve, adopte une position catégorique : il

20 Ibid.

21 Télégramme de M. Dejean, 26 novembre 1961, in Documents diplomatiques français, Paris, La Documentation française, 1998, p. 656-657.

22 Télégramme de M. Dejean, 29 décembre 1961, in ibid., p. 791-794. 
est du devoir de l'Occident d'aider la Finlande à renforcer sa neutralité, quitte à étendre ce statut à l'ensemble de la Scandinavie. Il est ainsi l'un des rares diplomates français dans les années 1960 à se montrer favorable au projet de Kekkonen visant à neutraliser toute cette région pour couper court à l'argument soviétique selon lequel une agression occidentale contre l'URSS se ferait forcément à travers la Scandinavie ${ }^{23}$.

Sans adhérer à ce projet, de Gaulle n'en soutient pas moins avec ferveur la neutralité de la Finlande : recevant Urho Kekkonen à Paris fin octobre 1962, en pleine crise des missiles cubains, il salue " la personnalité nationale " d'un " peuple qui a su s'établir, s'affermir et se maintenir en dépit de tant d'obstacles ${ }^{24}$. Dans les années qui suivent, et avec le relâchement des tensions, la perception française de la Finlande se fait au prisme du triptyque gaullien de "détente, entente, coopération ». En effet, de 1963 à 1969, de Gaulle cherche à restaurer l'influence perdue de la France via sa politique de dialogue avec Moscou, son éloignement des États-Unis et de l'OTAN et sa volonté de créer une Europe des États. L'idée d'indépendance vis-à-vis de toute entité susceptible de restreindre la souveraineté de la France devient le principe clé de la politique étrangère gaullienne. Celle-ci atteint son paroxysme dans les années 1965-1967 : les forces françaises sont retirées du commandement intégré de l'OTAN en mars 1966 ; la France multiplie les contacts avec les pays d'Europe orientale et, plus généralement, avec la plupart des États de la planète ; Paris n’hésite pas à remettre en question les équilibres communautaires au sein du Marché commun. L'objectif affiché n'est autre que de provoquer un mouvement général d'émancipation à l'égard des deux grands partout en Europe. Dans ces conditions, la diplomatie française porte une attention de plus en plus grande à la Finlande et à ses rapports avec le Kremlin, une attention qui atteint son paroxysme au cours de la décennie suivante mais qui trouve bel et bien ses origines dans la politique gaullienne d'indépendance.

Malgré tout, les Français restent méfiants quant aux usages que Moscou peut faire de la neutralité finlandaise et appellent le gouvernement d'Helsinki à rester prudent, notamment vis-à-vis de la nouvelle proposition soviétique de Conférence sur la sécurité européenne (CSE) destinée à consacrer le statu quo politique et territorial européen en proclamant l'intangibilité des frontières et le nonrecours à la force. La proposition, présentée par le Kremlin comme un moyen de pérenniser la détente, se situe dans la droite ligne des plans de neutralisation envisagés depuis les années $1950^{25}$. Les autorités françaises semblent d'autant plus circonspectes qu'au moment même où Moscou intensifie son offensive

23 Ibid.

24 Allocution et toast en l'honneur d'Urho Kekkonen et de son épouse, 24 octobre 1962, in Charles de Gaulle, Discours et messages, t. IV, 1962-1965, EDI8, 2014.

25 Télégramme d'O. Wormser, 4 février 1967. MAE, Europe 1966-1970, URSS, 2666. 
diplomatique pour faire accepter la CSE, les troupes du pacte de Varsovie interviennent en Tchécoslovaquie pour y réprimer le printemps de Prague : la volonté de l'URSS brejnévienne de préserver sa sphère d'influence européenne paraît inébranlable. Alors qu'en France de Gaulle quitte le pouvoir en 1969 et que sa politique d'indépendance, quasi sacralisée, est reprise par ses successeurs immédiats, le projet de CSE, conjugué au renouveau de la menace des Soviétiques sur leurs voisins, met la Finlande au premier plan des relations est-ouest, conduisant Paris et Helsinki à se rapprocher davantage.

\section{L'ENGAGEMENT EN FAVEUR DE LA CSCE, UN MOYEN DE SOUTENIR LA NEUTRALITÉ FINLANDAISE}

La répression du printemps de Prague par les troupes du pacte de Varsovie en août 1968 provoque un tournant dans les relations franco-finlandaises en ce qu'elle conduit la France, dirigée par Georges Pompidou à partir de 1969, à s'engager plus avant dans la défense de la précaire neutralité finlandaise. Cela se traduit par la nomination en 1969 à Helsinki de l'ambassadeur Gérard André, véritable artisan du rapprochement entre les deux pays. André joue un rôle central au moment de l'acceptation française du projet soviétique de CSE, jusque-là perçu comme de la pure propagande. Avec d'autres diplomates, il fait partie de ceux qui estiment nécessaire d'enrichir l'ordre du jour de cette conférence avec des thèmes - comme la coopération culturelle, le respect des droits de l'homme, l'échange d'observateurs pendant les manœuvres militaires ou le droit de chaque pays de conduire sa politique étrangère comme il l'entend - qui empêcheraient le Kremlin de réitérer à Helsinki ou à Belgrade ce qu'il a fait à Prague en $1968^{26}$.

On comprend en effet au Quai d'Orsay que la volonté de l'URSS de maintenir son glacis est telle que rien ne doit être négligé pour assurer la sécurité des nations directement menacées par Moscou. À Helsinki, Gérard André multiplie les contacts avec ses interlocuteurs finlandais et, de par les nombreux rapports sur la politique intérieure et extérieure finlandaise qu’il transmet à Paris, pousse la diplomatie française à s'engager davantage en faveur de la Finlande - que l'OTAN inclut en 1968 dans la "zone grise " sous influence soviétique ${ }^{27}$ - et de ses initiatives pour la sécurité européenne. Ainsi, lorsqu’en avril 1969 la plupart des Occidentaux voient la main de Moscou dans le mémorandum sur la tenue d'une CSE que diffuse le gouvernement de Kekkonen, André se montre, lui, convaincu qu'il s'agit bien d'une initiative finlandaise dans la mesure où, contrairement à ce que souhaite Leonid Brejnev, le mémorandum invite les États-Unis et le

26 Nicolas Badalassi, En finir avec la guerre froide, op. cit., p. 116-120.

27 Dépêche d'Helsinki, 11 décembre 1968. MAE, Europe 1961-1970, Finlande, 188QO/72. 
Canada à prendre part à la conférence, qui se déroulerait à Helsinki ${ }^{28}$. Il est clair, pour l'ambassadeur, qu'en agissant en faveur de la détente via la promotion de la CSE, la Finlande cherche une "reconnaissance publique de [sa] neutralité par les grandes puissances " et un renforcement de sa sécurité à l'égard de l'URSS ${ }^{29}$. Dans le même temps, proposer la CSE lui permet de justifier son absence de prise de position dans la querelle est-ouest à propos de la reconnaissance de la RDA ${ }^{30}$. En outre, si la CSE se tient à Helsinki, la Finlande sera par la force des choses à l'abri de toute entreprise de la part des participants : c'est là un argument de taille en faveur de l'acceptation de la CSE, argument repris par Pompidou lui-même ${ }^{31}$.

Le rapprochement franco-finlandais qui s'opère dans la première moitié de la décennie 1970 s'appuie également sur la volonté française de chercher une assurance contre les effets que pourrait provoquer l'élargissement de la CEE au Danemark et à la Norvège. Alors qu'André partage les craintes finlandaises qu'un tel élargissement n'accentue le clivage entre l'Est et l'Ouest, n'isole la Finlande et ne rompe l'équilibre précaire sur lequel est fondée la sauvegarde de son indépendance vis-à-vis de l'URSS ${ }^{32}$, le Quai d'Orsay et l'Élysée donnent des gages à Helsinki sur le maintien d'une solide coopération économique et commerciale avec la CEE, qui se concrétise avec la signature d'accords de libre-échange en 1972 et $1973^{33}$. Par ailleurs, la France exhorte les Finlandais à poursuivre leur engagement en faveur du projet Nordek destiné à réaliser l'intégration économique des pays nordiques : pour Paris, ce projet est une bonne chose dans la mesure où il confronte Moscou aux " parentés ethniques et linguistiques " de ces pays, qui partagent " un sentiment très vif d'une commune appartenance à une civilisation originale $»^{34}$. Lorsque Kekkonen suspend les négociations du Nordek en 1970, Gérard André explique aussitôt que le désir de ne pas provoquer le Kremlin est à l'origine de cette décision et que, dès lors, le soutien français à la CSE est plus nécessaire que jamais : la conférence pourrait renforcer la neutralité finlandaise, qu'il définit comme «la recherche d'un équilibre entre une volonté très ferme d'indépendance nationale et le souci de s'assurer en toutes circonstances la confiance du puissant voisin de l'Est $»^{35}$. Une CSE enrichie d'un volet sur le respect des droits de l'homme et proclamant l'inviolabilité des frontières permettrait de protéger

28 Télégramme d'Helsinki, 13 juin 1969. MAE, Europe 1961-1970, Finlande, 188QO/72.

29 Rapport de la sous-direction d'Europe occidentale (SDEO), 12 août 1970. MAE, Europe 19611970, Finlande, 188QO/72.

30 Dépêche d'Helsinki, 13 juin 1969. MAE, Europe 1961-1970, Finlande, 188QO/72.

31 Entretien Pompidou/Ceausescu, 15 juin 1970. Archives nationales de France - Pierrefitte-surSeine (AN), 5 AG 2 111, Roumanie.

32 Télégramme de G. André, 12 janvier 1970. MAE, Europe 1961-1970, Finlande, 188QO/72.

33 Télégramme de Paris à Helsinki, 12 janvier 1970. MAE, Europe 1961-1970, Finlande, 188QO/72.

34 Rapport de la SDEO, 24 août 1970. MAE, Europe 1961-1970, Suède, 205QO/80.

35 Télégramme de G. André, 6 avril 1970 ; Rapport de la SDEO, 12 août 1970. MAE, Europe 19611970, Finlande, 188QO/72. 
l'attachement des Finlandais au mode de vie occidental et à la démocratie tout en démontrant aux Soviétiques que le maintien de l'indépendance de la Finlande présente plus d'avantages que d'inconvénients ${ }^{36}$.

Si André parvient à convaincre le Quai d'Orsay, ses arguments en faveur de la Finlande n'ont aucun effet auprès des autorités militaires françaises qui, au contraire des responsables de la diplomatie, voient en la Finlande un pays soumis aux Soviétiques, ce que prouverait l'hésitation finlandaise à signer un accord avec la CEE. De fait, en matière militaire, la coopération franco-finlandaise est quasiment nulle. En 1972 puis de nouveau en 1974, la direction de l'Armement refuse de vendre à Helsinki les missiles antichar Milan en raison des atteintes au secret militaire que pourraient causer les affinités supposées entre l'armée finlandaise et l'Armée rouge ${ }^{37}$. Les efforts du Quai d'Orsay visant à convaincre l'étatmajor français que c'est précisément ce genre de refus qui rend la Finlande tributaire des armements soviétiques ne mènent à rien ${ }^{38}$ : pour la Défense française, la proximité fenno-soviétique n'encouragerait pas les alliés de la France, et notamment les États-Unis, à se porter acquéreurs de ces missiles à l'excellente réputation si Helsinki s'en procurait ${ }^{39}$. Pour André, un tel manque de bonne volonté rend la CSE plus urgente que jamais.

Au total, si l'engagement français en faveur de ce qui va devenir la Conférence pour la sécurité et la coopération en Europe (CSCE) s'explique aussi par le refus de Pompidou de demeurer à la remorque de Willy Brandt en matière de relations est-ouest, la volonté après les événements de Prague d'aider les neutres, et particulièrement la Finlande, à garantir leur sécurité face à Moscou est un facteur à ne pas négliger. Il ne faut donc pas décevoir les Finlandais, qui multiplient les efforts pour se soustraire de l'influence russe et qui, de plus, risquent de se sentir un peu seuls du fait de l'adhésion ou de l'association à la CEE de leurs voisins scandinaves $^{40}$. Ainsi, si CSCE il y a, dit Pompidou à Willy Brandt, elle devra en partie se tenir à Helsinki ${ }^{41}$.

Malgré cela, la diplomatie française reste prudente, suspectant toujours les Soviétiques de vouloir tirer les ficelles. Aussi les Français considèrent-ils qu'il ne faut pas faire d'Helsinki " la capitale de la sécurité européenne, car cette ville

36 Télégramme de G. André, 18 mars 1970. MAE, Europe 1961-1970, Finlande, 188QO/72.

37 Rapport de G. André, 10 août 1973 ; Rapport du chef d'escadrons Milliot, 3 août 1973. MAE, Europe 1971-1976, Finlande, 188QO/97.

38 Rapport du général Jean Simon, 25 septembre 1973. MAE, Europe 1971-1976, Finlande, 188QO/97.

39 Rapport, 8 janvier 1974. MAE, Europe 1971-1976, Finlande, 188QO/97.

40 Lettre du directeur d'Europe Claude Arnaud à G. André, 27 septembre 1971. MAE, Europe 1971-1976, Organismes internationaux et grandes questions internationales, 2922.

41 Entretien Pompidou/Brandt, 26 janvier 1971, Paris. AN, 5 AG 2 105, RFA. 
est excentrée géographiquement et politiquement $»^{42}$. Ils refusent ainsi l'idée de Moscou consistant à faire en sorte que la future CSCE accouche d'un organisme paneuropéen permanent de sécurité qui aurait son siège à Helsinki et veillerait au désarmement en Europe. Pour Pompidou, établir un tel organisme "dans un pays aussi sensible aux sollicitations soviétiques " tout comme étendre la neutralité en Europe par le biais du désarmement serait extrêmement dangereux ${ }^{43}$ : la détente politique doit être au préalable pérennisée, car la présence des armées en Europe centrale est l'une des conséquences de la tension est-ouest, pas l'une de ses causes. C'est précisément pour cette raison que le président français s'oppose aux MBFR (Mutual and Balanced Forces Reductions) négociées à Vienne dès 1973 et soutenues par les neutres : d'après lui, elles risquent d'aboutir à la neutralisation de l'ensemble de l'Europe centrale, Allemagne incluse ${ }^{44}$. Pompidou craint qu'une réduction des forces de l'OTAN et du pacte de Varsovie en Europe centrale ne favorise la «finlandisation » de l'Allemagne ${ }^{45}$.

Cette question de la dénucléarisation d'une partie de l'Europe est l'une des principales pierres d'achoppement entre la France et la Finlande, qui soutient les MBFR tout comme elle s'était prononcée en faveur des plans Khrouchtchev, Rapacki et Gomulka des années 1957-1964 destinés à dénucléariser la partie centrale du continent. De même, dans les années 1960 et 1970, les dirigeants français s'opposent au projet de Kekkonen de dénucléariser l'Europe du Nord : non seulement cela rendrait la zone vulnérable à la pression soviétique, mais cela pourrait compromettre la sécurité de tout le continent. Paris refuse également le " programme de désarmement pour l'Europe " proposé par Kekkonen aux Nations unies en octobre 1979, le jugeant contradictoire et dangereux ${ }^{46}$.

Mais si Paris et Helsinki ne s'entendent pas sur la question de la détente militaire, il en va tout autrement pour ce qui est de favoriser la détente politique et l'amélioration des échanges de toutes natures en Europe, notamment de part et d'autre du rideau de fer. La CSCE, qui se déroule à Helsinki et à Genève entre 1972 et 1975, vient conforter le sentiment exprimé à maintes reprises par la diplomatie française depuis le début de la guerre froide que la Finlande - ainsi que la Suède, la Suisse et l'Autriche -, malgré sa neutralité, est très attachée aux valeurs occidentales de démocratie et de libéralisme.

42 Note de la sous-direction d'Europe orientale, $1^{\text {er }}$ septembre 1970. MAE, Europe 1944-..., Finlande, 86.

43 Télégramme de G. de Courcel, 25 mai 1973. AN, 5 AG 2 1041, Relations est-ouest.

44 Entretien Pompidou/Nixon, 31 mai 1973. AN, 5 AG 2 1023, États-Unis.

45 Entretien Pompidou/Brandt, 10 février 1972. AN, 5 AG 2 106, RFA.

46 Rapport de la direction des Affaires stratégiques et du désarmement (DASD), 23 mai 1980. MAE, Europe 1977-1980, Finlande, 4411. 
À la CSCE, la collusion entre l'Ouest et les neutres prend un tour concret en raison des thèmes novateurs qui y sont abordés, à commencer par les droits de l'homme, la coopération culturelle et les contacts humains, que Moscou considère " bourgeois " et comme autant de prétextes de l'Ouest pour intervenir dans les affaires intérieures du bloc socialiste. Mais, en dépit des critiques de l'URSS, la Finlande défend les idées de l'Occident. Elle le fait cependant de façon subtile et sans provoquer les Soviétiques, en privilégiant les thèmes les moins offensifs comme la culture ou l'éducation. Cette approche est fort appréciée par les Français, qui procèdent de manière similaire pour ne pas braquer le Kremlin ${ }^{47}$.

Cette vision positive de la Finlande et de ses partenaires neutres a néanmoins ses limites et, sur certains thèmes, les divergences entre les Français et les représentants de ces pays conduisent à de véritables confrontations. Cela est notamment le cas lorsque Helsinki et Stockholm tentent de promouvoir le désarmement ou quand la France cherche à insérer dans l'Acte final d'Helsinki une clause relative à la protection des droits et responsabilités quadripartites sur l'Allemagne hérités de la conférence de Potsdam de 1945. Elle doit alors affronter les Suédois et d'autres délégations comme la Suisse ou la Yougoslavie, qui lui reprochent de créer, parmi les participants à la CSCE, une discrimination entre grandes et moyennes puissances $^{48}$. La France est ainsi accusée d'adopter une attitude qu'elle a toujours dénoncée.

\section{DU FRONT COMMUN POUR SAUVER LA DÉTENTE À LA FIN DE LA GUERRE FROIDE}

Lorsque la détente atteint ses limites après 1975, la France de Valéry Giscard d'Estaing tente contre vents et marées de maintenir la politique de rapprochement avec l'Est inaugurée par de Gaulle et de s'appuyer sur la détérioration progressive des relations soviéto-américaines pour faire valoir auprès du Kremlin sa volonté d'être un pont entre les deux blocs ${ }^{49}$.

C'est dans ce cadre que s'inscrit le désir ouvertement formulé par la diplomatie française de se rapprocher davantage des neutres nordiques, qui ont su prouver à la CSCE qu'ils étaient, comme Paris, très attachés à la fois à la détente et à la démocratie. Certes, note le Quai d'Orsay, la Suède et la Finlande voient d'un mauvais œil l'expansionnisme soviétique de la deuxième moitié des années 1970, mais ne peuvent pour autant se permettre de suivre les Américains dans leur offensive contre le communisme. En d'autres termes, ces pays sont sur la même

47 Télégramme de G. André, 21 décembre 1972. MAE, Europe 1971-1976, Organismes internationaux, 2925.

48 Télégrammes de Fernand-Laurent, 3 mars 1975. MAE, Europe 1971-1976, Organismes internationaux, 2927.

49 Rapport de la SDEO, 22 janvier 1980. MAE, Europe 1976-1980, Finlande, 4411. 
longueur d'onde que la France, du moins jusqu'à l'arrivée au pouvoir de François Mitterrand en 1981. Le fait de les soutenir dans leur politique de neutralité apparaît plus que jamais comme un moyen de manifester l'opposition française au "bloc-à-bloc " ${ }^{50}$. Par ailleurs, le rapprochement avec les neutres du Nord - dont le dynamisme en matière de lutte en faveur du désarmement est bien connu - vise également à promouvoir le projet français de conférence sur le désarmement en Europe lancé en 1978 et qui doit étendre les mesures de confiance prévues par la $\mathrm{CSCE}^{51}$. De la sorte, Paris court-circuiterait les projets finlandais sur le désarmement nucléaire ${ }^{52}$.

En parallèle, on est bien conscients en France que les initiatives de Moscou ne vont pas sans inquiéter la Finlande. En janvier 1978, le Quai d'Orsay note l'accroissement du potentiel militaire de l'URSS dans l'océan Arctique et, de fait, la dégradation des rapports entre Moscou et Oslo, ces deux facteurs étant susceptibles de menacer la sécurité finlandaise ${ }^{53}$. C'est donc à la fois pour les assurer de son amitié et de son soutien, pour souligner la continuité de sa politique mais aussi pour préparer les réunions de Belgrade et Madrid sur les suites de la CSCE - censées vérifier que les dispositions de l'Acte final d'Helsinki signé en 1975 sont correctement appliquées - que Giscard entend resserrer les liens avec les pays neutres du Nord. Alors que la guerre froide reprend de la vigueur, il effectue une visite d'État en Finlande en juin 1980 puis, le même mois, reçoit le couple royal suédois à Paris. La Finlande fait une fois encore l'objet d'une considération particulière du fait de son rôle durant le processus d'Helsinki, de sa position vis-à-vis de l'URSS et de ses efforts aux Nations unies en faveur du maintien de la paix ${ }^{54}$. Preuve en est que c'est Jacques Chazelle, figure de proue de la délégation française à la CSCE, qui est nommé ambassadeur à Helsinki en 1975.

En outre, le Quai d'Orsay souligne à plusieurs reprises que la Finlande est le seul pays neutre à ne pas condamner l'intervention en Afghanistan et que, contrairement à la Suède, elle a " la sagesse ", comme Giscard, de ne pas insister sur la question des droits de l'homme ${ }^{55}$. Le président français est en effet fort critique à l'égard de la moralpolitik de Jimmy Carter, qu'il juge contre-productive et

50 Rapport de la SDEO, 11 février 1980. MAE, Europe 1976-1980, Finlande, 4411.

51 Les mesures de confiance (MDC) visent à éviter les malentendus frontaliers et, pour les Occidentaux, à rendre plus difficile une intervention militaire de l'URSS sur le territoire de l'un de ses pays satellites. Les principales MDC concernent les échanges d'observateurs lors des manœuvres militaires et la notification des mouvements de troupes.

52 Rapport de la DASD, 23 mai 1980. MAE, Europe 1977-1980, Finlande, 4411.

53 Rapport de la sous-direction d'Europe orientale, 25 janvier 1978. MAE, Europe 1977-1980, Finlande, 4411.

54 Ibid.

55 Télégramme de J. Chazelle, 18 février 1980. MAE, Europe 1976-1980, Finlande, 4411. 
maladroite ${ }^{56}$. Intensifier les relations avec Helsinki est donc aussi un bon moyen pour la France de préserver l'estime de l'URSS. Les considérations économiques ne doivent pas non plus être négligées : les Français constatent en effet une dépendance économique accrue de la Finlande vis-à-vis de l'URSS à la suite du choc pétrolier de 1973 - qui a augmenté le prix du pétrole soviétique et réduit les marchés occidentaux - et en raison de la mise en œuvre progressive de l'accord passé entre Helsinki et le Comecon cette même année ${ }^{57}$. De fait, la diplomatie française entend contribuer à la diversification des importations finlandaises et cherche à pénétrer plus profondément le marché finlandais, en essayant notamment de vendre à Kekkonen le savoir-faire français en matière de technologie nucléaire civile $^{58}$. La coopération militaire franco-finlandaise reste, elle, très faible, malgré la relative évolution du point de vue des autorités militaires françaises à l'égard de la neutralité finlandaise : si, en 1975-1976, la France cherche à vendre à la Finlande l'avion d'entraînement Alpha-Jet, de fabrication franco-allemande, elle se heurte aux dispositions du traité de Paris de 1947, qui interdit à Helsinki d'acquérir du matériel aéronautique d'origine allemande ${ }^{59}$. En revanche, Paris prévoit en octobre 1979 l'envoi d'une mission civile et militaire à Helsinki afin d'évoquer avec les Finlandais les problèmes politico-stratégiques intéressant la Laponie finlandaise $^{60}$. Les archives ne disent pas si cette rencontre a effectivement lieu.

Quoi qu'il en soit, le tournant des années 1970 et 1980 inaugure un nouveau rapprochement entre la France et la Finlande qui trouvera sa consécration avec l'intégration de cette dernière au Marché commun en 1995. Cela ne se fera cependant pas sans accroc. À la veille des bouleversements de 1989, l'une des principales préoccupations françaises est d'empêcher la dilution de la Communauté dans une grande Europe. De fait, Mitterrand s'oppose à tout nouvel élargissement, notamment vers les pays de l'AELE que sont l'Autriche, la Suède et la Finlande - celle-ci devient membre à part entière de l'Association en 1986, au moment où la perestroïka gorbatchévienne assouplit la menace soviétique. La diplomatie française souhaite plutôt faire de ces pays une "zone tampon " autour des Douze de la CEE : les neutres devront être utilisés par la France et ses alliés comme instruments du rapprochement est-ouest à venir ; en conséquence, le refus français d'une adhésion au Marché commun est un signe manifeste de la volonté de Paris de ménager les Soviétiques, devant lesquels Mitterrand entend bien apparaître comme un partenaire bienveillant et privilégié. En outre, intégrer

56 Frédéric Bozo, La politique étrangère de la France, op. cit., p. 78.

57 Rapport de la SDEO, 3 mars 1977. MAE, Europe 1976-1980, Finlande, 4411.

58 Entretien Giscard d'Estaing/Kekkonen, 3 juin 1980, Helsinki. MAE, Europe 1976-1980, Finlande, 4411.

59 Rapport de G. André, 13 janvier 1975. MAE, Europe 1971-1976, Finlande, 188QO/97.

60 Télégramme de J. Chazelle, 24 octobre 1979. MAE, Europe 1976-1980, Finlande, 4411. 
ces États neutres dans la Communauté freinerait forcément le processus visant à l'établissement d'une politique de défense communautaire ${ }^{61}$.

Ainsi, lorsque le mur de Berlin tombe, la position de la France à l'égard des neutres reflète une fois de plus la politique étrangère du pays. L'attitude ambiguë de Mitterrand au moment de la fin de la guerre froide et de la réunification allemande se retrouve vis-à-vis de la Finlande et de la Suède : d'un côté, il est clair que ces pays aspirent à rejoindre le monde occidental alors triomphant, via notamment leur adhésion au Marché commun ; de l'autre, Paris cherche à les en maintenir éloignés afin qu'ils servent le projet mitterrandien consistant en un rapprochement en douceur des deux côtés du rideau de fer et qu'ils facilitent le dialogue entre Paris et Moscou. La France assure les neutres nordiques de son amitié et de son soutien, mais pas au point de sacrifier son statut et ses relations avec l'URSS ${ }^{62}$.

\section{Conclusion}

Au final, si la Finlande ne figure pas parmi les priorités de la politique étrangère de la France au cours de la guerre froide, la question de la neutralité finlandaise n'en suscite pas moins l'intérêt constant de Paris. Cet intérêt est directement lié à la perception française des rapports est-ouest. L'opposition précoce de la France à la logique bipolaire et son attachement aux principes de l'indépendance et du droit à la neutralité expliquent en grande partie ses manifestations de sympathie et de solidarité à l'égard d'une Finlande qui, dès 1948, tente de rester neutre pour satisfaire son voisin soviétique et éviter de tomber définitivement dans son escarcelle. La fragile position finlandaise provoque ainsi, du côté de la diplomatie française, débats et interrogations quant aux enjeux et à la viabilité de la neutralité dans l'Europe de la guerre froide. Plus encore, l'attention portée à la Finlande reflète systématiquement les préoccupations de la France en matière de sécurité et de coopération européenne, qu'il s'agisse de s'interroger sur l'opportunité ou non d'étendre la neutralité en Europe dans le cadre d'un règlement de la question allemande, de se rapprocher des neutres nordiques pour asseoir la politique gaullienne de "détente, entente et coopération ", ou de profiter des initiatives finlandaises pour combattre la politique des blocs et ouvrer au rapprochement des deux côtés du rideau de fer.

En d'autres termes, si le soutien à la neutralité finlandaise est empreint d'une indéniable sincérité, il apparaît surtout comme un moyen de faire valoir la politique étrangère française, notamment lorsqu'elle concerne la sécurité du

61 Frédéric Bozo, Mitterrand, la fin de la guerre froide et l'unification allemande. De Yalta à Maastricht, Paris, Odile Jacob, 2005, p. 98-100.

62 Sur la France et la fin de la guerre froide, voir ibid. 
continent, le désarmement, le sort de l'Allemagne, la construction européenne et, bien entendu, les relations avec le Kremlin. Plus largement, Paris affiche un soutien résolu aux neutres, mais à condition que cela ne nuise pas aux attributs de la puissance française.

La fin des années 1960 et la décennie 1970 apparaissent néanmoins comme le vrai tournant des relations entre la France et la Finlande dans la deuxième moitié du XXe siècle. Tandis que Paris cherche à pérenniser la politique gaullienne d'indépendance et que l'URSS multiplie les initiatives plus ou moins brutales en faveur de la consécration du statu quo européen, comme en témoigne l'intervention à Prague en 1968, la CSCE constitue une opportunité à la fois pour les Finlandais et les Français : aux premiers, elle permet de se poser en intermédiaires incontournables entre l'Est et l'Ouest tout en garantissant leur sécurité ; pour les seconds, elle est l'occasion de multilatéraliser les principes défendus par de Gaulle dans les années 1960 et notamment le droit pour chaque pays de conduire sa politique extérieure dans le sens qu'il souhaite. Ainsi, le désir d'indépendance de la France et celui de la Finlande de protéger sa neutralité se rejoignent au seuil de la décennie 1970 dans un combat commun contre l'interventionnisme soviétique et en faveur du libéralisme politique. La neutralité finlandaise semble alors atteindre ses limites : peut-on encore considérer la Finlande comme neutre alors même que la CSCE lui permet de prendre fait et cause pour l'ensemble des composantes du modèle occidental, qu'il s'agisse du respect de la démocratie et des droits de l'homme, de l'amélioration des échanges économiques, humains et culturels ou de la libre circulation de l'information ? L'événement qu'est la CSCE ne fait que révéler au grand jour des interrogations que les diplomates français en poste en Finlande et en Suède se posent depuis le début de la guerre froide : est-il possible d'être réellement neutre en période de guerre froide, dans la mesure où ce conflit est d'abord idéologique et se fonde sur le choix entre deux modèles de société ?

Les archives finlandaises pourraient certainement faire état des réflexions des responsables finlandais sur cette question fondamentale qui les concerne au premier chef, les documents français soulevant plus de questions qu'ils n'en résolvent. Le problème de l'élargissement des sources est en effet central et, finalement, les quelques éléments fournis ici ne laissent entrevoir qu'une maigre partie des relations et perceptions mutuelles franco-finlandaises après 1945. Le point de vue adopté étant simplement français, il offre une vision forcément restreinte des liens entre les deux pays. Des recherches approfondies dans les dépôts d'archives finlandais permettraient de dresser un panorama plus complet et, surtout, de voir comment les Finlandais présentent et utilisent leur statut de pays neutre dans leurs rapports avec la France. 